\title{
$R$-SEPARATION OF VARIABLES FOR THE FOUR-DIMENSIONAL FLAT SPACE LAPLACE AND HAMILTON-JACOBI EQUATIONS
}

\author{
BY
}

E. G. KALNINS AND WILLARD MILLER, JR. ${ }^{1}$

\begin{abstract}
All $R$-separable orthogonal coordinate systems for the complex equations $\sum_{i=1}^{4} \partial_{i i} \Psi=0$ and $\Sigma_{i-1}^{4}\left(\partial_{i} W\right)^{2}=0$ are classified and it is shown that these equations separate in exactly the same systems.
\end{abstract}

1. Introduction. We study the problem of $R$-separation of variables for the complex flat space Laplace and Hamilton-Jacobi equations

$$
\text { (a) } \sum_{i=1}^{4} \frac{\partial^{2} \Psi}{\partial\left(z^{i}\right)^{2}}=0 \text {, (b) } \sum_{i=1}^{4}\left(\frac{\partial W}{\partial z^{i}}\right)^{2}=0 \text {. }
$$

Here the complex metric is $d s^{2}=\sum_{i=1}^{4}\left(d z^{i}\right)^{2}$. We show that these two equations separate in precisely the same orthogonal coordinate systems and classify all possibilities. In particular, we show that the $R$-separable coordinates correspond to coordinates which permit pure separation for the Helmholtz equations on the manifolds $E_{4}$ (flat space), $S_{2} \times S_{2}, S_{3} \times E_{1}$ and $S_{4}$ where $S_{j}$ is the complex $j$-dimensional sphere. Detailed group theoretic classifications of separable coordinates on the first three manifolds appear in earlier papers by the authors [1], [2] while an analysis of $S_{4}$ will appear shortly [3]. It follows from these results that each $R$-separable system $\left\{x^{j}\right\}$ for (1.1)(a) is characterized by a triplet of second-order commuting symmetry operators $\left\{L_{1}, L_{2}, L_{3}\right\}$ in the enveloping algebra of $o(6, \mathrm{C})$, the symmetry algebra of this equation. The $R$-separable solutions $\Psi_{\alpha,}$ of (1.1)(a) corresponding to $\left\{x^{j}\right\}$ are characterized by the eigenvalue equations

$$
L_{i} \Psi_{\alpha_{j}}=\alpha_{i} \Psi_{\alpha_{j}}, \quad i=1,2,3,
$$

where $\alpha_{1}, \alpha_{2}, \alpha_{3}$ are the separation constants [4]. This relationship between $o(6, \mathrm{C})$ and separation of variables for (1.1)(a) permits use of group representation theory to derive properties of the $R$-separated solutions [5].

The problem of orthogonal $R$-separation for (1.1)(a) was treated in 1905 by

Received by the editors June 7, 1977.

AMS (MOS) subject classifications (1970). Primary 22E70, 33A75, 35A25, 53B20.

Key words and phrases. Conformal symmetry, flat space, Hamilton-Jacobi equation, Laplace equation, separation of variables.

${ }^{1}$ Supported in part by NSF Grant MCS 76-04838-A01. 
Bôcher [6] who constructed all systems whose coordinate surfaces are families of confocal cyclides. As we shall show, Bôcher's method actually suffices to construct all orthogonal $R$-separable systems for (1.1)(a). However, Bôcher's method is very special and does not permit one to conclude that all separable coordinate systems have been found. In this paper, based partially on techniques of Eisenhart [7] we develop a more general method which allows us to obtain an exhaustive list of separable systems for (1.1)(a), each of which has a simple geometric and group theoretic interpretation. Moreover, we are able to establish the equivalence of separation for (1.1)(a) and (1.1)(b). Similar comments hold for the distinct real forms of these complex equations, e.g., the real Laplace and wave equations with their Hamilton-Jacobi counterparts. One need only modify the results obtained here by classifying the possible real metrics with the appropriate signature. In particular, our results show that the lists of orthogonal $R$-separable coordinates for the wave equation found in [1], [2], [4] are complete.

In $\$ 2$ we examine the problem of $R$-separation for the Laplace and Hamilton-Jacobi equations in arbitrary $n$-dimensional Riemannian spaces and prove some lemmas which clear up a number of obscurities and ambiguities which have appeared in the recent literature devoted to separation of variables. In $\$ 3$ we prove that for $n=4$ every conformally flat metric in Stäckel form is conformal to a Stäckel form metric which satisfies the Robertson condition. This result is used in $\$ 4$ to find a complete list of such metrics and obtain the principal results announced above.

For the singular case $n=3$, corresponding results were obtained in [8]. We expect the techniques and results of this paper to extend to the case $n>4$ and to some nonflat metrics. In another paper we will classify the nonorthogonal $R$-separable coordinate systems for (1.1).

The equations and coordinate systems treated in this paper are of great interest for applications because of their close connection with the wave equation and the Hamilton-Jacobi equations of classical and relativistic physics. In particular, most of the special functions of mathematical physics arise as solutions of (1.1)(a) via separation of variables and many of their properties can be obtained from the representation theory of $o(6, C)$ (15], [9D).

2. $R$-separation. We begin by examining the general problem of $R$-separation for the Laplace and Hamilton-Jacobi equations

$$
\begin{aligned}
& \text { (a) } \Delta_{2} \Psi=\frac{1}{\sqrt{g}} \sum_{i, j=1}^{n} \partial_{x^{\prime}}\left(\sqrt{g} g^{i j \partial_{x^{\prime}}} \Psi\right)=0, \\
& \text { (b) } \Delta_{1} W=\sum_{i, j=1}^{n} g^{i j \partial_{x^{\prime}} W \partial_{x^{\prime}} W=0 .}
\end{aligned}
$$

Here, $d s^{2}=\Sigma g_{i j} d x^{i} d x^{j}$ is a complex Riemannian metric, $g=\operatorname{det}\left(g_{i j}\right) \neq 0$, 
$\sum_{j} g^{i j} g_{j k}=\delta_{k}^{i}$, and $g_{i j}=g_{j i}$. We restrict our attention to orthogonal coordinates

$$
d s^{2}=\sum_{j} H_{j}^{2}\left(d x^{j}\right)^{2}=\sum_{i j} g_{i j} d x^{i} d x^{j} .
$$

By $R$-separation of (2.1)(a) we mean that the assumption

$$
\Psi=M\left(x^{1}, \ldots, x^{n}\right) \prod_{j=1}^{n} A_{j}\left(x^{j}\right)
$$

permits the separation of this equation into $n$ ordinary differential equations, one for each factor $A_{j}$. Similarly, separation of (2.1)(b) means that the assumption

$$
W=\sum_{j=1}^{n} B_{j}\left(x^{j}\right)
$$

permits the separation of the Hamilton-Jacobi equation into $n$ ordinary differential equations.

(2.1)(a) has been studied by Moon and Spencer [10] who find that necessary and sufficient conditions for $R$-separability are (1) that the metric take the form

$$
H_{i}^{2}=\left(S / M_{i 1}\right) Q, \quad i=1, \ldots, n,
$$

where $S$ is a Stäckel determinant, $S=\operatorname{det} \Phi, \Phi=\left(\Phi_{i j}\right)$ where $\Phi_{i j}=\Phi_{i j}\left(x^{i}\right)$, $M_{i 1}$ is the $(i, 1)$ - cofactor of the Stäckel matrix $\Phi$, and $Q\left(x^{1}, \ldots, x^{n}\right)$ is an arbitrary function, (2) that

$$
\frac{H M^{2}}{Q S}=\prod_{j=1}^{n} f_{j}\left(x^{j}\right), \quad H=H_{1}, \ldots, H_{n},
$$

and (3) that $M$ satisfy

$$
\sum_{j=1}^{n} \frac{M_{j 1}}{S f_{j}} \partial_{x^{\prime}}\left(f_{j} \partial_{x^{\prime}} M^{-1}\right)+\alpha M^{-1}=0
$$

where $\alpha$ is a constant.

Conditions (2.2), (2.3) are obviously essential. However, the requirement that $\alpha$ be a constant in (2.4), while not necessarily incorrect, is rather misleading. In fact, a closer analysis of the $R$-separation problem for (2.1)(a) shows that it is necessary and sufficient for $\alpha$ to be of the more general form

$$
\alpha\left(x^{1}, \ldots, x^{n}\right)=\sum_{j=1}^{n} \frac{Q l_{j}\left(x^{j}\right)}{H_{j}^{2}}+c_{1}, \quad c_{1} \in \mathbf{C} .
$$

In this case the function $\hat{\Psi}=\prod_{i=1}^{n} A_{i}\left(x^{i}\right)$ satisfies 


$$
\sum_{j=1}^{n}\left[\frac{M_{i 1}}{S f_{j}} \partial_{x^{j}}\left(f_{j} \partial_{x^{\prime}} \hat{\Psi}\right)+\frac{Q l_{j}}{H_{j}^{2}} \hat{\Psi}\right]+c_{1} \hat{\Psi}=0
$$

an equation which separates into ordinary differential equations

$$
\frac{1}{f_{j}} \frac{d}{d x^{j}}\left(f_{j} \frac{d A_{j}}{d x^{j}}\right)+l_{j} A_{j}+\sum_{i=1}^{n} c_{i} \Phi_{j i} A_{j}=0, \quad j=1, \ldots, n,
$$

where $c_{2}, \ldots, c_{n}$ are the separation constants.

On the other hand the multiplier $M$ associated with a given $R$-separable coordinate system $\left\{x^{1}, \ldots, x^{n}\right\}$ is not unique. We say that two nonzero functions $M, \tilde{M}$ are equivalent if there exist nonzero functions $p_{j}\left(x^{j}\right), j=$ $1, \ldots, n$, such that $\tilde{M}=p_{1}^{-1}\left(x^{1}\right) \cdots p_{n}^{-1}\left(x^{n}\right) M$. Then $\Psi=M A_{1} \cdots A_{n}=$ $\tilde{M} \tilde{A}_{1}, \ldots, \tilde{A}_{n}$ where $\tilde{A}_{j}=p_{j} A_{j}$ so $\Psi$ is $R$-separable with multiplier $M$ if and only if it is $R$-separable with multiplier $\tilde{M}$. Note that $\tilde{M}$ satisfies (2.3)-(2.5) with $f_{j}, l_{j}$ replaced by

$$
\tilde{f}_{j}=p_{j}^{-2} f_{j}, \quad \tilde{l}_{j}=l_{j}+f_{j}^{\prime} p_{j}\left(p_{j}^{-1}\right)^{\prime} / f_{j}+p_{j}\left(p_{j}^{-1}\right)^{\prime \prime} .
$$

It follows that we can always choose the functions $p_{j}$ such that $\tilde{l}_{j} \equiv 0$ for $j=1, \ldots, n$; hence $M$ is equivalent to a multiplier $\tilde{M}$ for which $\alpha$ in (2.5) is a constant. Thus the criterion for $R$-separability as given in [10] can be saved, although one thereby loses the flexibility due to equivalence transforms.

There is another degree of freedom in the description of $R$-separation due to the fact that the characterization (2.2) of the metric in terms of Stäckel determinants may not be unique. Suppose $d s^{2}$ is a metric and $M$ a function satisfying (2.2)-(2.5), hence defining an $R$-separation of $(2.1)(a)$ in the coordinates $\left\{x^{1}, \ldots, x^{n}\right\}$ and let $\tilde{\Phi}$ be a Stäckel matrix in these coordinates, $\tilde{S}=\operatorname{det} \tilde{\Phi}$, and $\tilde{Q}$ a function such that

$$
H_{i}^{2}=\tilde{S} \tilde{Q} / \tilde{M}_{i 1},
$$

where $\tilde{M}_{i 1}$ is the $(i, 1)$-cofactor of $\tilde{\Phi}$.

LEMMA 1. The Stäckel matrix $\tilde{\Phi}$ determines an $R$-separation in the variables $\left\{x^{1}, \ldots, x^{n}\right\}$ with multiplier $\tilde{M}$ equivalent to $M$.

Proof. Comparing (2.2) and (2.9) we have $\tilde{Q} \tilde{S} /(Q S)=\tilde{M}_{i 1} / M_{i 1}$ for $i=$ $1, \ldots, n$. Since $M_{i 1}, \tilde{M}_{i 1}$ are independent of $x^{i}$ we have $Q S=k \tilde{Q} \tilde{S}$ where $k$ is a nonzero constant. By renormalization of $\tilde{Q}$ we can assume without loss of generality that $k=1$. It follows immediately that $M_{i 1}=\tilde{M}_{i 1}$ for all $i$. From (2.3), if the matrix $\tilde{\Phi}$ is to determine $R$-separation with multiplier $\tilde{M}$ then $\tilde{M}$ must satisfy

$$
H \tilde{M}^{2} / \tilde{Q} \tilde{S}=\prod_{j=1}^{n} \tilde{f}_{j}\left(x^{j}\right)
$$


for some nonzero functions $\tilde{f}_{j}$. (2.3) and (2.10) then imply

$$
\prod_{j=1}^{n}\left(\frac{f_{j}}{\tilde{f}_{j}}\right)=\frac{M^{2}}{\tilde{M}^{2}}
$$

Thus $\tilde{M}$ is equivalent to $M$ and it is enough to establish the lemma for $\tilde{M}=M$.

Expanding $S$ in cofactors we have

$$
S=\sum \Phi_{i 1} M_{i 1}=\sum \Phi_{i 1} \tilde{M}_{i 1}
$$

so the identity $Q S=\tilde{Q} \tilde{S}$ leads to

$$
\frac{\tilde{Q}}{Q}=\sum_{j=1}^{n} \Phi_{j 1}\left(x^{j}\right) \frac{\tilde{Q}}{H_{j}^{2}} .
$$

To show that $\tilde{M}=M$ determines an $R$-separation corresponding to (2.9) we must establish (2.4) for $S$ replaced by $\tilde{S}$ and $\alpha$ by a corresponding function $\tilde{\alpha}$. Now

$$
\begin{aligned}
& \sum \frac{M_{j 1}}{f_{j} \tilde{S}} \partial_{x^{\prime}}\left(f_{j} \partial_{x^{\prime}} M^{-1}\right)=\frac{\tilde{Q}}{Q} \sum \frac{M_{j 1}}{f_{j} S} \partial_{x^{\prime}}\left(f_{j^{\prime}} \partial_{x^{\prime}} M^{-1}\right) \\
& \quad=-\frac{\tilde{Q}}{Q}\left(\sum \frac{Q l_{j}}{H_{j}^{2}}+c_{1}\right) M^{-1}=-\sum \frac{\tilde{Q}}{H_{j}^{2}}\left(l_{j}+c_{1} \Phi_{j 1}\right) M^{-1}
\end{aligned}
$$

Thus

$$
\tilde{\alpha}=\sum_{j=1}^{n} \frac{\tilde{Q}}{H_{j}^{2}}\left(l_{j}\left(x^{j}\right)+c_{1} \Phi_{j 1}\left(x^{j}\right)\right) \text {. Q.E.D. }
$$

The above result shows that if a coordinate system is $R$-separable then any Stäckel matrix $\tilde{\Phi}$ satisfying (2.9) can be used to define the separation.

It is well known [11], [12] that a necessary and sufficient condition that (2.1)(b) be separable in the coordinates $\left\{x^{1}, \ldots, x^{n}\right\}$ is (2.2). Thus every $R$-separable system for (2.1)(a) also separates (2.1)(b) but the converse is not generally true.

It is also well known [12] that a necessary and sufficient condition for separability $(M \equiv 1)$ of the Helmholtz equation $\hat{\Delta}_{2} \Theta=E \Theta, E \neq 0$, corresponding to the metric $d \hat{s}=\sum_{j=1}^{n} h_{j}^{2}\left(d x^{j}\right)^{2}$ is

$$
h_{i}^{2}=\frac{S}{M_{i 1}}, \quad \frac{h}{S}=\prod_{j=1}^{n} \hat{f}_{j}\left(x^{j}\right), \quad h=h_{1}, \ldots, h_{n},
$$

i.e., that $d \hat{s}^{2}$ be in Stäckel form and that the Robertson condition be satisfied. Eisenhart [12] has shown that the Robertson condition is equivalent to the 
requirement $R_{i j}=0$ for $i \neq j$ where $R_{i j}$ is the Ricci tensor with respect to the metric $d \hat{s}^{2}$.

The following result will prove useful.

LEMMA 2. Let $\left\{x^{1}, \ldots, x^{n}\right\}$ be an $R$-separable system for (2.1)(a)such that $d s^{2}=\Sigma_{j} H_{j}^{2}\left(d x^{j}\right)^{2}$ where $H_{j}^{2}=Q h_{j}^{2}$ and $d s^{2}=\Sigma_{j} h_{j}^{2}\left(d x^{j}\right)^{2}$ satisfies conditions (2.12). Then the multiplier can be chosen to be $M=Q^{(2-n) / 4}$. Furthermore

$$
\begin{gathered}
\hat{\Delta}_{2} M^{-1}+\alpha M^{-1}=0, \quad \alpha=\sum_{j=1}^{n} \frac{l_{j}\left(x^{j}\right)}{h_{j}^{2}}+c_{1}, \\
\hat{\Delta}_{2} \hat{\Psi}+\alpha \hat{\Psi}=0
\end{gathered}
$$

where $\Psi=M \hat{\Psi}$.

Proof. From (2.12) we have

$$
\frac{H}{Q S}=\frac{Q^{(n-2) / 2} h}{S}=Q^{(n-2) / 2} \prod_{j=1}^{n} \hat{f}_{j}\left(x^{j}\right)
$$

whereas (2.3) yields

$$
\frac{H}{Q S}=M^{-2} \prod_{j=1}^{n} f_{j}\left(x^{j}\right) \text {. }
$$

Thus $M$ is equivalent to the multiplier $Q^{(2-n) / 4}$. Q.E.D.

Note from (2.14) that $\hat{\Psi}$ satisfies a "Helmholtz equation with separable potential", hence it can be completely separated: $\hat{\Psi}=\Pi_{j=1}^{n} A\left(x^{j}\right)$.

LEMMa 3. Suppose $d \hat{s}^{2}=\Sigma h_{j}^{2}\left(d x^{j}\right)^{2}$ satisfies conditions (2.12) and that $H_{j}^{2}=$ $Q h_{j}^{2}$. Then (2.1)(a) is $R$-separable corresponding to the system $d s^{2}=\Sigma H_{j}^{2}\left(d x^{j}\right)^{2}$ if and only if $M=Q^{(2-n) / 4}$ satisfies (2.13).

As an example of the application of Lemmas 2 and 3 we consider the notion of $R$-separation of the flat space Helmholtz equation

$$
\sum_{i=1}^{n} \frac{\partial^{2}}{\partial\left(z^{i}\right)^{2}} \Psi=E \Psi, \quad E \neq 0,
$$

as introduced by Moon and Spencer [13]. These authors show that a necessary condition for $R$-separation of $(2.15)$ in the coordinates $\left\{x^{j}\right\}$ is that the metric satisfy (2.2) and (2.3) with $Q \equiv 1$. However, the above lemmas then imply that (since $R_{i j}=0$ for flat space) we can choose the multiplier $M$ so $M \equiv 1$. Thus $\left\{x^{j}\right\}$ permits $R$-separation of $(2.15)$ iff it permits pure separation. It follows that $R$-separation for (2.15), and any Helmholtz equation for an Einstein space, is a useless concept.

3. The Robertson condition. We shall now restrict our attention to (2.1)(a) for which $d s^{2}=\Sigma H_{j}^{2}\left(d x^{j}\right)^{2}$ is a flat space metric, i.e., the complex Laplace. 
equation in $n$ variables. Then condition (2.2) for $R$-separation implies $d s^{2}=$ $Q d \hat{s}^{2}$ where $d \hat{s}^{2}=\Sigma h_{j}^{2}\left(d x^{j}\right)^{2}, h_{j}=S / M_{j 1}$. Thus $d \hat{s}^{2}$ is in Stäckel form and corresponds to a conformally flat space. To solve the separation of variables problem for the Laplace equation one must necessarily study these conformally flat metrics in Stäckel form. In [7] Eisenhart has investigated these metrics and we list some of his formulas which are relevant to our study.

The condition that $d \hat{s}^{2}$ be in Stäckel form is

$$
\begin{aligned}
\frac{\partial^{2} \ln h_{i}^{2}}{\partial x^{j} \partial x^{k}}-\frac{\partial \ln h_{i}^{2}}{\partial x^{j}} \frac{\partial \ln h_{i}^{2}}{\partial x^{k}}+\frac{\partial \ln h_{i}^{2}}{\partial x^{j}} \frac{\partial \ln h_{j}^{2}}{\partial x^{k}}+\frac{\partial \ln h_{i}^{2}}{\partial x^{k}} \frac{\partial \ln h_{k}^{2}}{\partial x^{j}}=0, \\
\quad(j \neq k) .
\end{aligned}
$$

In consequence of (3.1) the components of the Riemann curvature tensor for $i, j, k$ different may be written

$$
R_{j i i k}=\frac{3}{4} h_{i}^{2} \partial^{2} \ln h_{i}^{2} / \partial x^{j} \partial x^{k}
$$

and the nondiagonal elements of the Ricci tensor are

$$
R_{j k}=\sum_{l=1}^{n} \frac{1}{h_{l}^{2}} R_{j l l k}=\frac{3}{4} \frac{\partial^{2} \ln \Pi^{\prime} h_{l}^{2}}{\partial x^{j} \partial x^{k}}
$$

where $\Pi^{\prime}$ indicates the product of the $h$ 's except $h_{j}$ and $h_{k}$. For $n \geqslant 4$ the necessary and sufficient condition that $d \hat{s}^{2}$ be conformally flat is that the conformal curvature tensor $C_{l i j k}$ identically vanish [14], i.e.,

$$
\begin{aligned}
C_{l i j k}= & R_{l i j k}+\frac{1}{n-2}\left(\hat{g}_{j l} R_{i k}-\hat{g}_{l k} R_{i j}+\hat{g}_{i k} R_{l j}-\hat{g}_{i j} R_{l k}\right) \\
& +\frac{R}{(n-1)(n-2)}\left(\hat{g}_{l k} \hat{g}_{i j}-\hat{g}_{l j} \hat{g}_{i k}\right)=0, \quad \hat{g}_{j l}=h_{j}^{2} \delta_{j l} .
\end{aligned}
$$

Given the metric $d \hat{s}^{2}$ the function $Q=e^{2 \lambda}$ is determined by solving the equations

$$
\lambda_{i j}=(n-2)^{-1}\left(R \hat{g}_{i j} / 2(n-1)-R_{i j}\right)-\frac{1}{2} \hat{g}_{i j} \Delta_{1} \lambda
$$

where

$$
\lambda_{i j}=\lambda_{, i j}-\lambda_{, i} \lambda_{j}, \quad \lambda_{, i}=\partial \lambda / \partial x^{i}
$$

and $\lambda_{, i j}$ is a second covariant derivative of $\lambda$ with respect to $\hat{g}_{k l}$.

For $n=3$ it was shown explicitly in [8] that by suitable renormalization of $Q$ it is always possible to choose $d \hat{s}^{2}$ such that $R_{i j}=0$ for $i \neq j$. For $n=4$ we have

THEOREM 1. Let $d \hat{s}^{2}=\sum_{j=1}^{4} h_{j}^{2}\left(d x^{j}\right)^{2}$ be a conformally flat metric in Stäckel form. Then there exists a nonzero function $q$ such that $d \hat{s}^{2}=q d \tilde{s}^{2}=$ 
$\sum q \tilde{h}_{j}^{2}\left(d_{i}^{j}\right)^{2}$ where the metric $d \tilde{s}^{2}$ is in Stäckel form and such that $\boldsymbol{R}_{i j}=0$ for $i \neq j$.

Our proof of this result is rather complicated and will be accomplished through a series of lemmas valid for all $n>3$, some of which are interesting in their own right.

LeMma 4. Let d $\hat{s}^{2}=\sum_{j=1}^{n} h_{j}^{2}\left(d x^{j}\right)^{2}$ be conformally flat and in Stäckel form. Then there exist nonzero functions $X_{j}\left(x^{j}\right), \Psi_{j k}\left(x^{j}, x^{k}\right)=-\Psi_{k j}, f\left(x^{1}, \ldots, x^{n}\right)$ such that

$$
\begin{gathered}
h_{j}^{2}=X_{j} f \prod_{k \neq j} \Psi_{j k}, \quad j=1, \ldots, n, \\
(\mathrm{a}) \partial_{j k}\left(\Psi_{j k} f\right)=0, \quad(\mathrm{~b}) \partial_{j k}\left(\frac{\Psi_{j k}}{\Psi_{i j} \Psi_{i k}}\right)=0 \quad(i, j, k \neq) .
\end{gathered}
$$

$\left(\right.$ Here $\left.\partial^{2} f / \partial x^{j} \partial x^{k}=\partial_{j k} f.\right)$

Proof. From (3.1) for $i=k$ we find

$$
\partial_{i j} \ln \left(h_{i}^{2} / h_{j}^{2}\right)=0, \quad i \neq j,
$$

so

$$
h_{i}^{2}=\varphi_{i j} \Theta_{i j}, \quad h_{j}^{2}=\varphi_{j i} \Theta_{i j},
$$

where $\varphi_{i j}, \varphi_{j i}, \Theta_{i j}$ are functions such that $\partial_{j} \varphi_{i j}=\partial_{i} \varphi_{j i}=0$. The condition $C_{j i i k}=0(j, i, k \neq)$ implies

$$
R_{j i i k}=\left(h_{i}^{2} /(n-2)\right) R_{j k} .
$$

From (3.2) and (3.3) we find

$$
\partial_{j k} \ln \left(\Pi^{\prime} h_{l}^{2} / h_{i}^{2(n-2)}\right)=0, \text { so } \partial_{j k} \ln \left(h_{l}^{2} / h_{i}^{2}\right)=0
$$

for $i, l$ distinct from $j, k$. It follows that

$$
h_{i}^{2}=\prod_{l \neq i, j} \Psi_{i l}\left(x^{i}, x^{l}\right) \Theta_{i j}, \quad h_{j}^{2}=\prod_{l \neq i, j} \Psi_{j l}\left(x^{j}, x^{l}\right) \Theta_{i j}
$$

These results are consistent for all values of $i$ and $j$ only if (3.6) is valid. Substituting (3.0) into (3.1) we obtain the conditions (3.7)(a), (b). Q.E.D.

LEMMA 5. Let $d \hat{s}^{2}=\sum_{j=1}^{n} h_{j}^{2}\left(d x^{j}\right)^{2}$ be in Stäckel form. Then $d \hat{s}^{2}=e^{2 \varphi} d \hat{s}^{2}$ is in Stäckel form iff

$$
\partial_{j k} \varphi+2 \partial_{j} \varphi \partial_{k} \varphi+\partial_{j} \varphi \partial_{k} \ln h_{j}^{2}+\partial_{k} \varphi \partial_{j} \ln h_{k}^{2}=0
$$

for all unequal $j, k$. The nondiagonal elements of the corresponding Ricci tensors are related by

$$
\tilde{R}_{j k}=\hat{R}_{j k}+\frac{3}{2}(n-2) \partial_{j k} \varphi \quad(j, k \neq) .
$$


Proof. (3.9) follows directly from (3.1), and (3.10) is a consequence of identity (28.6) in [14]. Q.E.D.

Clearly, Theorem 1 will follow from Lemma 5 provided we can find a function $\varphi$ such that

$$
\hat{R}_{j k}+\frac{3}{2}(n-2) \partial_{j k} \varphi=0, \quad(j, k \neq) .
$$

Eisenhart [12] has shown that the nondiagonal elements of the Ricci tensor for a metric in Stäckel form are given by

$$
\hat{R}_{j k}=\frac{3}{4} \partial_{j k} \ln \Pi^{\prime} h_{i}^{2} .
$$

Thus if $d \hat{s}^{2}$ satisfies the hypotheses of Lemma 4 then (3.11) requires

$$
\partial_{j k} \varphi=-\frac{1}{2} \partial_{j k} \ln f
$$

or

$$
e^{2 \varphi}=f^{-1} t_{1} t_{2} \cdots t_{n}
$$

where $t_{i}=t_{i}\left(x^{i}\right)$ is a nonzero function. The transformed metric $d \tilde{s}^{2}$ has the form

$$
\tilde{h}_{j}^{2}=X_{j} t_{1} \cdots t_{n} \prod_{l \neq j} \Psi_{j l}
$$

The condition that (3.14) be in Stäckel form, i.e., the condition that $e^{2 \varphi}$ preserves Stäckel form is easily determined from (3.7) or (3.9).

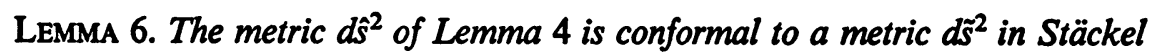
form which satisfies the Robertson condition iff there exist nonzero functions $t_{i}\left(x^{i}\right), i=1, \ldots, n$, such that

$$
\partial_{j k}\left(t_{j} t_{k} \Psi_{j k}\right)=0 \quad(j \neq k) .
$$

To complete the proof of Theorem 1 we will show that if $\left\{\Psi_{a b}=-\Psi_{b a}\right\}$ is a set of functions satisfying (3.7)(b) then there exist nonzero functions $\left\{t_{i}\right\}$ such that (3.15) holds for $n=4$. Let $i, j, k$ be three distinct indices. Then (3.7)(b) is equivalent to the existence of functions $\Theta_{a b}\left(x^{a}, x^{b}\right)$ such that

$$
\begin{aligned}
& \Psi_{j k}=\Theta_{i j} \Psi_{i k}+\Theta_{i k} \Psi_{j i}, \\
& \Psi_{i k}=\Theta_{j i} \Psi_{j k}+\Theta_{j k} \Psi_{i j}, \\
& \Psi_{i j}=\Theta_{k i} \Psi_{k j}+\Theta_{k j} \Psi_{i k} .
\end{aligned}
$$

These relations imply the equalities

$$
\begin{aligned}
& \text { (a) }\left(\Theta_{i j} \Theta_{j i}-1\right) \Psi_{j k}=\left(\Theta_{i k}-\Theta_{i j} \Theta_{j k}\right) \Psi_{i j}, \\
& \text { (b) }\left(\Theta_{k j} \Theta_{j i}-\Theta_{k i}\right) \Psi_{j k}=\left(\Theta_{k j} \Theta_{j k}-1\right) \Psi_{j i}, \\
& \text { (c) }\left(\Theta_{i j} \Theta_{k i}-\Theta_{k j}\right) \Psi_{i k}=\left(\Theta_{k i} \Theta_{i k}-1\right) \Psi_{i j} .
\end{aligned}
$$


The functions $\Theta_{a b}$ are not uniquely determined since, for example, the functions

$$
\Theta_{j i}^{\prime}=\Theta_{j i}+h\left(x^{j}\right) \Psi_{i j}, \quad \Theta_{j k}^{\prime}=\Theta_{j k}-h\left(x^{j}\right) \Psi_{j k}
$$

also satisfy (3.16). Now fix $x^{i}=x_{0}^{i}$ such that the $\left\{\Psi_{a b}\right\}_{x^{\prime}=x_{0}^{i}}$ are all nonzero and normalize the functions $\left\{\Theta_{a b}\right\}$ such that $\Theta_{i j} \Theta_{j i}=1$. It follows from (3.17)(a) that $\Theta_{j k}=\Theta_{i k} / \Theta_{i j}$. Similarly for fixed $x^{i}=x_{0}^{i}$ we can normalize so that $\Theta_{k i} \Theta_{i k}=1$, thus (3.17)(c) implies $\Theta_{k j}=\Theta_{i j} \Theta_{k i}$. We conclude that $\Theta_{k j}=$ $\Theta_{j k}^{-1}$, independent of $x^{i}$. Then (3.17)(b) implies $\Theta_{k j}=\Theta_{k i} / \Theta_{j i}$ for all $x^{i}$. We have now determined functions such that the last two equations of (3.16) hold for all $x^{i}$. Defining $\Theta_{i j}=\Theta_{j i}^{-1}, \Theta_{i k}=\Theta_{k i}^{-1}$ for all $x^{i}$ we can verify that the first equation of (3.16) is also valid. It follows easily that there exist nonzero functions $t_{a}=t_{a}\left(x^{a}\right)$ such that $\Theta_{i k}^{-1}=-t_{k} / t_{i}, \Theta_{i j}^{-1}=-t_{j} / t_{i}$, so

$$
t_{i}^{-1} \Psi_{j k}+t_{j}^{-1} \Psi_{i k}+t_{k}^{-1} \Psi_{j i}=0 .
$$

Thus there exist functions $b_{a}, a=i, j, k$, such that

$$
\begin{aligned}
& \text { (a) } \Psi_{i j}=b_{i} t_{j}^{-1}-b_{j} t_{i}^{-1}=\left(t_{i} t_{j}\right)^{-1}\left(c_{i}-c_{j}\right), \\
& \text { (b) } \Psi_{j k}=b_{j} t_{k}^{-1}-b_{k} t_{j}^{-1}=\left(t_{j} t_{k}\right)^{-1}\left(c_{j}-c_{k}\right) \text {, } \\
& \text { (c) } \Psi_{i k}=b_{k} t_{i}^{-1}-b_{i} t_{k}^{-1}=\left(t_{i} t_{k}\right)^{-1}\left(c_{k}-c_{i}\right),
\end{aligned}
$$

where $c_{a}=b_{a} t_{a}$. Again the functions $b_{a}$ and $c_{a}$ are highly nonunique since it is only the differences $c_{i}-c_{j}$ (independent of $x^{k}$ ), etc. which appear in (3.19). By addition of an appropriate function we can assume $c_{i}=c_{i}\left(x^{l}\right)$. Then (3.19)(a), (c) imply $c_{j}=c_{j}\left(x^{i}, x^{j}\right), c_{k}=c_{k}\left(x^{i}, x^{k}\right)$. From (3.19)(b) we have $\partial_{i}\left(c_{j}-c_{k}\right)=0$ so

$$
c_{j}\left(x^{i}, x^{j}\right)-c_{j}\left(x_{0}^{i}, x^{j}\right)=c_{k}\left(x^{i}, x^{k}\right)-c_{k}\left(x_{0}^{i}, x^{k}\right)=h\left(x^{i}\right) .
$$

By adding $-h\left(x^{i}\right)$ to all functions $c_{a}$ we get new functions $\tilde{c}_{a}=\tilde{c}_{a}\left(x^{a}\right)$ which also satisfy (3.19). Thus $\partial_{j k}\left(t_{j} t_{k} \Psi_{j k}\right)=0$, etc.

Now suppose $\partial_{i j} \ln \Psi_{i j} \neq 0$ for some $\Psi_{i j}$. Then $\partial_{i} c_{i} \neq 0, \partial_{j} c_{j} \neq 0$. Next consider the functions $\Psi_{i j}, \Psi_{j l}, \Psi_{i l}$ for $l \neq i, j, k$. Arguing as above we find .nonzero functions $s_{a}=s\left(x^{a}\right)$, and functions $d_{a}=d_{a}\left(x^{a}\right), a=i, j, l$, such that

$$
s_{i} s_{j} \Psi_{i j}=d_{i}-d_{j}, \quad s_{j} s_{l} \Psi_{j l}=d_{j}-d_{l}, \quad s_{i} s_{l} \Psi_{i l}=d_{l}-d_{i} .
$$

Since $d_{i}$ and $d_{j}$ are nonconstant we can compare expressions (3.19) and (3.20) for $\Psi_{i j}$ to obtain (to within trivial multiplications and additions by scalars) either $t_{a}=s_{a}, c_{a}=d_{a}$ or $t_{a} / s_{a}=1 / d_{a}, c_{a}=-1 / d_{a}, a=i, j$. If $d_{l}$ is nonconstant we easily obtain a uniform representation of the six functions $\Psi_{\alpha \beta}$ in the form $t_{\alpha} t_{\beta} \Psi_{\alpha \beta}=c_{\alpha}-c_{\beta}$. If, however, $\partial_{l} d_{l}=0$ then we have relations of the form $t_{j} s_{l} \Psi_{j l}=h_{l}, t_{i} s_{l} \Psi_{i l}=h_{l}, h_{l}=h_{l}\left(x^{l}\right)$ which do not assume the appearance (3.19). Still, setting $t_{l}=s_{l} h_{l}^{-1}$ we find 


$$
\partial_{j l}\left(t_{j} t_{l} \Psi_{j l}\right)=\partial_{i l}\left(t_{i} t_{l} \Psi_{i l}\right)=0 .
$$

Now consider $\Psi_{k l}$. Treating $\Psi_{i k}, \Psi_{i l}, \Psi_{k l}$ and reasoning as above we see that there exist nonzero functions $u_{a}=u_{a}\left(x^{a}\right)$ such that

$$
u_{l} \Psi_{i k}+u_{k} \Psi_{i l}+u_{i} \Psi_{k l}=0 \text {. }
$$

If $\partial_{l} d_{l} \neq 0$ then

$$
t_{i} t_{k} \Psi_{i k}=c_{k}-c_{i}, \quad t_{i} t_{l} \Psi_{i l}=c_{i}-c_{l}
$$

so

$$
\begin{aligned}
t_{k} t_{l} \Psi_{k l} & =-\left(t_{k} t_{l} / u_{i}\right)\left(u_{l} \Psi_{i k}+u_{k} \Psi_{i l}\right) \\
& =\left(c_{i} / u_{i} t_{i}\right)\left(u_{l} t_{l}-u_{k} t_{k}\right)+\left(1 / u_{i} t_{i}\right)\left(c_{l} u_{k} t_{k}-c_{k} u_{l} t_{l}\right) .
\end{aligned}
$$

Since $\partial_{i}\left(t_{k} t_{l} \Psi_{k l}\right)=0$ we see that if $\partial_{i}\left(u_{i} t_{i}\right) \neq 0$ then there exists a constant $\alpha$ such that

$$
c_{l} u_{k} t_{k}-c_{k} u_{l} t_{l}=\alpha\left(u_{l} t_{l}-u_{k} t_{k}\right)
$$

Thus

$$
\partial_{k l}\left(t_{k} t_{l} \Psi_{k l}\right)=0 .
$$

If, however, $u_{i} t_{i}=\beta=$ const then since $\partial_{i} c_{i} \neq 0$ we have $u_{l} t_{l}=u_{k} t_{k}=$ const, so again (3.22) holds.

Now suppose $\partial_{l} d_{l}=0$. Then

$$
t_{i} t_{k} \Psi_{i k}=c_{k}-c_{i}, \quad t_{i} t_{l} \Psi_{i l}=1
$$

so

$$
t_{k} t_{l} \Psi_{k l}=\left(c_{i} / u_{i} t_{i}\right) t_{l} u_{l}-\left(1 / u_{i} t_{i}\right)\left(c_{k} t_{l} u_{l}+t_{k} u_{k}\right) .
$$

If $\partial_{i}\left(u_{i} t_{i}\right) \neq 0$ then there exists a constant $\alpha$ such that

$$
c_{k} t_{l} u_{l}+t_{k} u_{k}=\alpha t_{l} u_{l}
$$

and (3.22) holds. If $u_{i} t_{i}=\beta$ then since $\partial_{i} c_{i} \neq 0$ we have $t_{l} u_{l}=0$, which is impossible. Thus (3.22) holds in all cases.

For $n=4$ the only remaining possibility is that $\partial_{a b} \ln \Psi_{a b}=0$, for all $a$, $b=i, j, k, l, a \neq b$. Then $\Psi_{a b}=A^{a b}\left(x^{a}\right) B^{a b}\left(x^{b}\right)$. A case-by-case analysis employing (3.7)(b) and much simpler than the above shows that there always exist nonzero functions $t_{a}\left(x^{a}\right), a=i, j, k, l$, such that $\partial_{a b}\left(t_{a} t_{b} \Psi_{a b}\right)=0$. Indeed if $\Psi_{j k}=A\left(x^{j}\right) B\left(x^{k}\right)$ with $\partial_{j} A \neq 0, \partial_{k} B \neq 0$ it is easy to show that the $t_{a}$ can be chosen so that $t_{j}=A^{-1}, t_{k}=B^{-1}$. If each $\Psi_{a b}$ is a nontrivial function of at most one variable then we can choose $t_{1}=\cdots=t_{4}=1$. This completes the proof of Theorem 1 for $n=4$.

LEMMA 7. For $n>4$ let $d s^{2}=\sum_{j=1}^{n} H_{j}^{2}\left(d s^{j}\right)^{2}$ be a flat space metric such that $d s^{2}=Q d \hat{s}^{2}$ where $d \hat{s}^{2}=\sum_{j-1}^{n} h_{j}^{2}\left(d x^{j}\right)^{2}$ is in Stäckel form and satisfies the Robertson condition. Then the coordinates $\left\{x^{1}, \ldots, x^{n}\right\}$ permit $R$-separation for the flat space Laplace equation $\Delta_{2} \Psi=0$ iff 


$$
R=\sum_{j=1}^{n} \frac{l_{j}\left(x^{j}\right)}{h_{j}^{2}}+c
$$

where $R$ is the scalar curvature corresponding to the metric $d \hat{s}^{2}$.

Proof. By Lemmas 2 and 3 the coordinates $\left\{x^{k}\right\}$ permit $R$-separation iff $\hat{\Delta}_{2} Q^{(n-2) / 4}+\alpha Q^{(n-2) / 4}=0$ where

$$
\alpha=\sum_{j=1}^{n} \frac{\tilde{l}_{j}\left(x^{j}\right)}{h_{j}^{2}}+c_{1},
$$

and $\hat{\Delta}_{2}$ is the Laplace-Beltrami operator corresponding to the metric $d \hat{s}^{2}$. Since $d \hat{s}^{2}$ is conformally flat (3.5) holds for $Q=e^{2 \sigma}$. Multiplying both sides of (3.5) by $h_{j}^{-2}$, setting $i=j$ and summing on $j$ we readily obtain

$$
\hat{\Delta}_{2} Q^{(n-2) / 4}+\frac{(n-2)}{4(n-1)} R Q^{(n-2) / 4}=0 \text {. Q.E.D. }
$$

THEOREM 2. Let $\left\{x^{k}\right\}$ be a coordinate system for four-dimensional flat space with metric $d s^{2}=\sum_{j=1}^{4} H_{j}^{2}\left(d x^{j}\right)^{2}$. Then $\left\{x^{k}\right\}$ permits $R$-separation of variables for the flat space Laplace equation iff $d s^{2}$ is conformal to a metric $d \hat{s}^{2}=$ $\sum_{j=1}^{4} h_{j}^{2}\left(d x^{j}\right)^{2}$ such that (1) $d \hat{s}^{2}$ is in Stäckel form, (2) d $\hat{s}^{2}$ satisfies the Robertson condition $R_{i j}=0(i \neq j)$, and (3) the scalar curvature of $d \hat{s}^{2}$ can be expressed in the form (3.24).

It follows from Lemma 1 that the validity of condition (3) is independent of which metric $d \hat{s}^{2}$ is chosen satisfying conditions (1) and (2). Thus to find all $R$-separable systems for the flat space Laplace equation it is sufficient to compute all conformally flat metrics $d \hat{s}^{2}$ which satisfy conditions (1) and (2), and then compute the scalar curvature of each to determine if condition (3) is satisfied.

Due to the crucial importance of Theorems 1 and 2 in this paper we indicate an alternate proof of these results. Let the metric $d \hat{s}^{2}$ satisfy the hypotheses of Theorem 1. From Lemmas 4 and 5 it follows easily that $d \tilde{s}^{2}=h_{1}^{-2} d \hat{s}^{2}=\sum_{i=1}^{4} H_{i}^{2}\left(d x^{i}\right)^{2}$ is also in Stäckel form where

$$
H_{1}^{2}=1, \quad H_{j}^{2}=q^{j}\left(x^{1}\right) \Psi_{1 j}^{2}\left(T_{1 j}^{j k}+T_{1 k}^{j k}\right)\left(T_{1 j}^{j l}+T_{1 l}^{j l}\right),
$$

$j, k, l=2,3,4$ are distinct, and $T_{1 j}, \Psi_{1 j}$ are functions of $\left\{x^{1}, x^{j}\right\}$ alone. Indeed the trick of multiplying by $h_{1}^{-2}$ allows us to take $f=\left[\Psi_{12} \Psi_{13} \Psi_{14}\right]^{-1}$ in Lemma 5 and (3.26) follows immediately from (3.7)(b) with $i=1$.

Now, fixing the variable $x^{1}$, we observe that the metric $H_{2}^{2}\left(d x^{2}\right)^{2}+$ $H_{3}^{2}\left(d x^{3}\right)^{2}+H_{4}^{2}\left(d x^{4}\right)^{2}$ in $\left\{x^{2}, x^{3}, x^{4}\right\}$ satisfies the Stäckel conditions (3.1) as well as the Robertson condition for metrics in three variables. These are precisely the conditions Eisenhart used to classify the possible Stäckel forms 
in three variables [12]. He found that the forms were of four types, the most complicated of which is

$$
H_{j}^{2}=\left(T_{1 j}-T_{1 k}\right)\left(T_{1 j}-T_{1 l}\right) \Psi_{1 j}^{2}, \quad j=2,3,4,
$$

where $T_{1 j}$ is a function of $\left\{x^{1}, x^{j}\right\}$ alone. (Here we must modify Eisenhart's results by allowing each function to depend on $x^{1}$.) Substituting (3.27) into (3.26) and imposing the remaining conditions (3.7)(b) for $i=2,3,4$, we can compute all possibilities for $T_{1 j}, \Psi_{1 j}$. One such solution is

$$
H_{j}^{2}=\frac{\left(\sigma_{j}-\sigma_{k}\right)\left(\sigma_{j}-\sigma_{l}\right)}{\left(\sigma_{1}-\sigma_{k}\right)\left(\sigma_{1}-\sigma_{l}\right)} q\left(x^{1}\right), \quad j, k, l=2,3,4,
$$

where $\sigma_{i}=\sigma_{i}\left(x^{i}\right)$. Multiplying $d \tilde{s}^{2}$ by $\left(\sigma_{1}-\sigma_{j}\right)\left(\sigma_{1}-\sigma_{k}\right)\left(\sigma_{1}-\sigma_{l}\right)$ and absorbing $q\left(x^{1}\right)$ through a renormalization of $x^{1}$ we obtain the metric

$$
\hat{H}_{i}^{2}=\left(\sigma_{i}-\sigma_{j}\right)\left(\sigma_{i}-\sigma_{k}\right)\left(\sigma_{i}-\sigma_{l}\right), \quad i, j, k, l=1,2,3,4,
$$

which is conformal to $d \hat{s}^{2}$, in Stäckel form, and satisfies the Robertson condition. A similar case-by-case study employing Eisenhart's other three standard forms in three variables leads in a straightforward manner to the exhaustive list (4.1)-(4.7) and verifies Theorem 1.

4. The classification of separable systems. Here we implement the results of Theorem 2 by constructing (for $n=4$ ) all conformally flat metrics $d \hat{s}^{2}=$ $\Sigma h_{j}^{2}\left(d x^{j}\right)^{2}$ which are in Stäckel form and satisfy the Robertson condition $R_{i j}=0, i \neq j$. It is a straightforward application of Eisenhart's results in [12] to classify all metrics $d \hat{s}^{2}$ which are (1) in Stäckel form, (2) satisfy the Robertson condition and (3) satisfy (3.8).

The possibilities are

$$
\begin{aligned}
& \text { [1] } h_{1}^{2}=X_{1}\left(\sigma_{1}-\sigma_{2}\right), \quad h_{2}^{2}=X_{2}\left(\sigma_{1}-\sigma_{2}\right) \text {, } \\
& h_{3}^{2}=X_{3}\left(\sigma_{3}-\sigma_{4}\right), \quad h_{4}^{2}=X_{4}\left(\sigma_{3}-\sigma_{4}\right),
\end{aligned}
$$

where the functions $X_{i}, \sigma_{i}$ depend on $x^{i}$ only,

$$
\begin{array}{cll}
\text { [2] } h_{1}^{2}=X_{1}\left(\sigma_{1}-\sigma_{2}\right), & h_{2}^{2}=X_{2}\left(\sigma_{1}-\sigma_{2}\right), \\
h_{3}^{2}=X_{3} \sigma_{1} \sigma_{2}\left(\sigma_{3}-\sigma_{4}\right), & h_{4}^{2}=X_{4} \sigma_{1} \sigma_{2}\left(\sigma_{3}-\sigma_{4}\right), \\
\text { [3] } h_{1}^{2}=\sigma_{2} \sigma_{3} \sigma_{4}, & h_{2}^{2}=X_{2}\left(\sigma_{23}+\sigma_{32}\right)\left(\sigma_{24}+\sigma_{42}\right) \\
h_{3}^{2}=X_{3}\left(\sigma_{32}+\sigma_{23}\right)\left(\sigma_{34}+\sigma_{43}\right), & h_{4}^{2}=X_{4}\left(\sigma_{42}+\sigma_{24}\right)\left(\sigma_{43}+\sigma_{34}\right),
\end{array}
$$

where $\sigma_{i j}$ is a function of $x^{i}$ alone,

$$
\text { [4] } h_{i}^{2}=X_{i}\left(\sigma_{i}-\sigma_{j}\right)\left(\sigma_{i}-\sigma_{k}\right)\left(\sigma_{i}-\sigma_{l}\right),
$$

with $i, j, k, l$ distinct, 


$$
\begin{aligned}
& \text { [5] } \quad h_{1}^{2}=X_{1}, h_{2}^{2}=\varphi\left(x^{1}\right) X_{2}\left(\sigma_{23}+\sigma_{32}\right)\left(\sigma_{24}+\sigma_{42}\right) \text {, } \\
& h_{3}^{2}=\varphi\left(x^{1}\right) X_{3}\left(\sigma_{32}+\sigma_{23}\right)\left(\sigma_{34}+\sigma_{43}\right) \text {, } \\
& h_{4}^{2}=\varphi\left(x^{1}\right) X_{4}\left(\sigma_{42}+\sigma_{24}\right)\left(\sigma_{43}+\sigma_{34}\right) \text {, } \\
& \text { [6] } h_{1}^{2}=X_{1}, \quad h_{2}^{2}=X_{2} f\left(x^{1}\right) \text {, } \\
& h_{3}^{2}=X_{3} \varphi\left(x^{1}\right)\left(\sigma_{3}-\sigma_{4}\right), \quad h_{4}^{2}=X_{4} \varphi\left(x^{1}\right)\left(\sigma_{3}-\sigma_{4}\right) \text {, } \\
& \text { [7] } h_{1}^{2}=X_{1}, \quad h_{2}^{2}=X_{2} f\left(x^{1}\right), \quad h_{3}^{2}=X_{3} g\left(x^{1}\right) \text {, } \\
& h_{4}^{2}=X_{4} h\left(x^{1}\right) \text {. }
\end{aligned}
$$

We now examine the remaining conditions imposed by the requirement

$$
C_{j i j j}=R_{j i j j}-\frac{1}{2}\left(h_{j}^{2} R_{i i}+h_{i}^{2} R_{i j}\right)+\frac{1}{6} R h_{j}^{2} h_{i}^{2}=0
$$

for $i \neq j$. Using the relations

$$
R_{i i}=\sum_{k} h_{k}^{-2} R_{k i i k}, \quad R=\sum_{l} h_{l}^{-2} R_{l l}
$$

we see that (4.8) is equivalent to

$$
\begin{aligned}
2 h_{k}^{2} h_{l}^{2} R_{j i j j}+2 h_{j}^{2} h_{i}^{2} R_{k l l k}= & h_{j}^{2} h_{k}^{2} R_{i l l i}+h_{j}^{2} h_{l}^{2} R_{i k k d} \\
& +h_{i}^{2} h_{k}^{2} R_{j l l}+h_{i}^{2} h_{l}^{2} R_{j k k j}
\end{aligned}
$$

where $i, j, k, l$ are distinct. Writing

$$
B_{k l j i}=h_{k}^{2} h_{l}^{2} R_{j i j}+h_{j}^{2} h_{i}^{2} R_{k l l k}
$$

we can express (4.9) in the form

$$
2 B_{k l, j t}=B_{k j, i l}+B_{k i, j} \text {. }
$$

Permuting $\{i, j, k, l\}$ we obtain two further conditions of this form and the three conditions are readily seen to be equivalent to

$$
B_{k l, j i}=B_{k j, i l}=B_{k i, j \cdot}
$$

We will substitute the various differential forms [1] $[7]$ into (4.11). Once the possible conformally flat metrics $d \hat{s}^{2}$ have been determined we will compute the functions $Q=e^{2 \lambda}$ from (3.5).

We first consider metrics of type [1]. If all the $\sigma_{i}$ are not constants then the metric may be taken in the form

$$
\begin{array}{ll}
h_{1}^{2}=X_{1}\left(x^{1}-x^{2}\right), & h_{2}^{2}=X_{2}\left(x^{1}-x^{2}\right), \\
h_{3}^{2}=X_{3}\left(x^{3}-x^{4}\right), & h_{4}^{2}=X_{4}\left(x^{3}-x^{4}\right),
\end{array}
$$

i.e., we can set $\sigma_{i}=x^{i}$ for all $i$. The condition $B_{12,34}=0$ is equivalent to 


$$
\begin{aligned}
& \left(x^{3}-x^{4}\right)^{3}\left[-2\left(\frac{1}{X_{1}}+\frac{1}{X_{2}}\right)+\left(x^{1}-x^{2}\right)\left\{\left(\frac{1}{X_{1}}\right)^{\prime}-\left(\frac{1}{X_{2}}\right)^{\prime}\right\}\right] \\
& +\left(x^{1}-x^{2}\right)^{3}\left[-2\left(\frac{1}{X_{3}}+\frac{1}{X_{4}}\right)+\left(x^{3}-x^{4}\right)\left\{\left(\frac{1}{X_{3}}\right)^{\prime}-\left(\frac{1}{X_{4}}\right)^{\prime}\right\}\right]=0 .
\end{aligned}
$$

Differentiating this equation successively with respect to $x^{1}$ we get that $\left(1 / X_{1}\right)^{(4)}=0$ so

$$
\frac{1}{X_{1}}=f\left(x^{1}\right)=a\left(x^{1}\right)^{3}+b\left(x^{1}\right)^{2}+c x^{1}+d ;
$$

similarly, $\left(X_{2}\right)^{-1}=-f\left(x^{2}\right)$. An analogous computation yields

$$
\frac{1}{X_{3}}=h\left(x^{3}\right)=-a\left(x^{3}\right)^{3}-e\left(x^{3}\right)^{2}-g x^{3}-k, \quad \frac{1}{X_{4}}=-h\left(x^{4}\right)
$$

If $a \neq 0$ the metric takes the form

$$
\begin{array}{r}
d s^{2}=\frac{\left(x^{1}-x^{2}\right)}{4}\left[\frac{\left(d x^{1}\right)^{2}}{\left(x^{1}-a_{1}\right)\left(x^{1}-a_{2}\right)\left(x^{1}-a_{3}\right)}\right. \\
-\frac{\left(x^{3}-x^{4}\right)}{4}\left[\frac{\left(d x^{2}\right)^{2}}{\left(x^{2}-a_{1}\right)\left(x^{2}-a_{2}\right)\left(x^{2}-a_{3}\right)}\right] \\
\left.-\frac{\left.\left(d x^{3}\right)^{2}\right)\left(x^{3}-b_{2}\right)\left(x^{3}-b_{3}\right)}{\left(x^{4}-b_{1}\right)\left(x^{4}-b_{2}\right)\left(x^{4}-b_{3}\right)}\right]
\end{array}
$$

This metric corresponds to the choice of elliptic type coordinates related to the reduction $o(6, \mathrm{C}) \supset o(3, \mathrm{C}) \times o(3, \mathrm{C})$ of the symmetry algebra $o(6, \mathrm{C})$ of the Laplace equation. If the roots $\left\{a_{i}\right\}$ are distinct and the roots $\left\{b_{i}\right\}$ are distinct we may take $a_{1}=a, b_{1}=b, a_{2}=b_{2}=1, a_{3}=b_{3}=0$. The corresponding four space coordinates can be chosen as

$$
\begin{array}{ll}
z^{1}=\frac{\xi_{2}}{\xi_{1}+i \xi_{3}}, & z^{2}=\frac{\zeta_{1}}{\xi_{1}+i \xi_{3}}, \\
z^{3}=\frac{\zeta_{2}}{\xi_{1}+i \xi_{3}}, & z^{4}=\frac{\zeta_{3}}{\xi_{1}+i \xi_{3}},
\end{array}
$$

where $\zeta_{1}^{2}+\zeta_{2}^{2}+\zeta_{3}^{2}=1$ and $\xi_{1}^{2}+\xi_{2}^{2}+\xi_{3}^{2}=-1$. In each case we have 
chosen Lamé type coordinates on the two-sphere $S_{2}$ according to

$$
\begin{array}{ll}
\zeta_{1}^{2}=\frac{x^{1} x^{2}}{a}, \quad \zeta_{2}^{2}=\frac{-\left(x^{1}-1\right)\left(x^{2}-1\right)}{a-1}, \quad \zeta_{3}^{2}=\frac{\left(x^{1}-a\right)\left(x^{2}-a\right)}{a(a-1)}, \\
\xi_{1}^{2}=\frac{-x^{3} x^{4}}{b}, \quad \xi_{2}^{2}=\frac{\left(x^{3}-1\right)\left(x^{4}-1\right)}{b-1}, \quad \xi_{3}^{2}=\frac{\left(x^{3}-b\right)\left(x^{4}-b\right)}{b(b-1)} .
\end{array}
$$

The function $Q$ is given by

$$
Q=\left[\sqrt{-x^{3} x^{4} / b}+\sqrt{-\left(x^{3}-b\right)\left(x^{4}-b\right) / b(b-1)}\right]^{-2}
$$

Note that the Riemann space is $S_{2} \times S_{2}$.

When some of the roots are equal in (4.14) the coordinates still take the form (4.16) but $\zeta$ and $\xi$ are now chosen as other separable systems on the two-spheres of radii 1 and $i$ respectively [4].

If, alternatively, $a=0$ in (4.14) the metric can be taken in the form

$$
\begin{aligned}
d \hat{s}^{2} & =\frac{\left(x^{1}-x^{2}\right)}{4}\left[\frac{\left(d x^{1}\right)^{2}}{\left(x^{1}-a_{1}\right)\left(x^{1}-a_{2}\right)}-\frac{\left(d x^{2}\right)^{2}}{\left(x^{2}-a_{1}\right)\left(x^{2}-a_{2}\right)}\right] \\
& +c^{2} \frac{\left(x^{3}-x^{4}\right)}{4}\left[\frac{\left(d x^{3}\right)^{2}}{\left(x^{3}-b_{1}\right)\left(x^{3}-b_{2}\right)}-\frac{\left(d x^{4}\right)^{2}}{\left(x^{4}-b_{1}\right)\left(x^{4}-b_{2}\right)}\right] .
\end{aligned}
$$

This metric corresponds to the choice of elliptic coordinates related to the reduction $o(6, \mathrm{C}) \supset \mathcal{E}(2, \mathrm{C}) \times \mathcal{E}(2, \mathrm{C})$. If the roots $\left\{a_{i}\right\}$ and $\left\{b_{i}\right\}$ are distinct we may take $a_{1}=b_{1}=1, a_{2}=b_{2}=0$. The four space coordinates can be chosen as

$$
\begin{aligned}
& z^{1}=Q^{1 / 2}\left[-\left(1-\left(x^{1}\right)^{2}\right)\left(1-\left(x^{2}\right)^{2}\right)\right]^{1 / 2}, \quad z^{2}=Q^{1 / 2} x^{1} x^{2} \\
& z^{3}=c Q^{1 / 2}\left[-\left(1-\left(x^{3}\right)^{2}\right)\left(1-\left(x^{4}\right)^{2}\right)\right]^{1 / 2}, \quad z^{4}=c Q^{1 / 2} x^{3} x^{4}
\end{aligned}
$$

where

$$
Q=1 \text { or } Q=\left[\left(x^{1}\right)^{2}+\left(x^{2}\right)^{2}-1+c^{2}\left(\left(x^{3}\right)^{2}-\left(x^{4}\right)^{2}-1\right)\right]^{-2} \text {. }
$$

(Here the two choices of $Q$ lead to conformally equivalent coordinates.) For general choices of $\left\{a_{i}\right\}$ and $\left\{b_{i}\right\}$ the coordinates $z^{i}$ are of the form

$$
z^{1}=Q^{1 / 2} x, \quad z^{2}=Q^{1 / 2} y, \quad z^{3}=Q^{1 / 2} \bar{x}, \quad z^{4}=Q^{1 / 2} \bar{y},
$$

where $(x, y)$ and $(\bar{x}, \bar{y})$ are chosen from among the various inequivalent elliptic coordinate systems in the complex Euclidean plane for which the flat 
space Helmholtz equation separates [2]. Here $Q$ is either 1 or $\left[x^{2}+y^{2}+\bar{x}^{2}\right.$ $\left.+\bar{y}^{2}\right]^{-2}$. The case $Q=1$ corresponds to flat space $E_{4}$.

If we allow any of the functions $\sigma_{i}$ in [1] to be constant it is straightforward to show that we just get special limiting cases of the coordinate systems already discussed. We refer the reader to the literature [2], [4] for lists of these systems.

Systems corresponding to metric [2] are conformally equivalent to metric [1] systems. This can be shown by dividing the metric coefficients by $\sigma_{1} \sigma_{2}$ and absorbing this factor into $Q$.

For metrics of type [3], if all the functions $\sigma_{i}$ are nonconstant the metric coefficients can be chosen as

$$
\begin{aligned}
& h_{1}^{2}=x^{2} x^{3} x^{4}, \quad h_{2}^{2}=X_{2}\left(x^{2}-x^{3}\right)\left(x^{2}-x^{4}\right), \\
& h_{3}^{2}=X_{3}\left(x^{3}-x^{2}\right)\left(x^{3}-x^{4}\right), \quad h_{4}^{2}=X_{4}\left(x^{4}-x^{2}\right)\left(x^{4}-x^{3}\right) .
\end{aligned}
$$

Making the transformation $x^{i} \rightarrow\left(x^{i}\right)^{-1}, i=2,3,4$, and extracting the factor $1 / x^{2} x^{3} x^{4}$ we obtain the new metric

$$
\begin{aligned}
& h_{1}^{2}=1, \quad h_{2}^{2}=X_{2}^{\prime}\left(x^{2}-x^{3}\right)\left(x^{2}-x^{4}\right), \\
& h_{3}^{2}=X_{3}^{\prime}\left(x^{3}-x^{2}\right)\left(x^{3}-x^{4}\right), \quad h_{4}^{2}=X_{4}^{\prime}\left(x^{4}-x^{2}\right)\left(x^{4}-x^{3}\right)
\end{aligned}
$$

where $X_{i}^{\prime}$ are new functions of the variables $x^{i}$. It is also readily seen that if the $\sigma_{i}$ are nonconstant for a metric of type [5] then this metric can also be reduced to (4.22). Dropping the primes in (4.22) we now proceed to evaluate the possible functions $X_{i}$. Substituting (4.22) into $B_{34,12}=B_{23,14}$ and dividing by $x^{2}-x^{4}$ we obtain the condition

$$
\begin{aligned}
& \left(x^{2}-x^{4}\right)^{3}\left[\left(x^{3}-x^{4}\right)\left(x^{3}-x^{2}\right)\left(\frac{1}{X_{3}}\right)^{\prime}+2\left(x^{2}+x^{4}-2 x^{3}\right)\left(\frac{1}{X_{3}}\right)\right] \\
& +\left(x^{4}-x^{3}\right)^{3}\left[\left(x^{2}-x^{3}\right)\left(x^{2}-x^{4}\right)\left(\frac{1}{X_{2}}\right)^{\prime}+2\left(x^{3}+x^{4}-2 x^{2}\right)\left(\frac{1}{X_{2}}\right)\right] \\
& +\left(x^{3}-x^{2}\right)^{3}\left[\left(x^{4}-x^{2}\right)\left(x^{4}-x^{3}\right)\left(\frac{1}{X_{4}}\right)^{\prime}+2\left(x^{2}+x^{3}-2 x^{4}\right)\left(\frac{1}{X_{4}}\right)\right]=0 .
\end{aligned}
$$

Differentiating this equation twice with respect to $x^{3}, x^{4}$ and four times with respect to $x^{2}$ we find $\left(X_{2}^{-1}\right)^{(5)}=0$ so

$$
\frac{1}{X_{2}}=f\left(x^{2}\right)=\alpha\left(x^{2}\right)^{4}+\beta\left(x^{2}\right)^{3}+\gamma\left(x^{2}\right)^{2}+\delta x^{2}+e,
$$

and in general $X_{i}^{-1}=f\left(x^{i}\right), i=2,3,4$. If $\alpha \neq 0$ we have the metric 


$$
\begin{aligned}
d \hat{s}^{2}=\left(d x^{1}\right)^{2}+\sum_{i=2}^{4} \frac{\alpha^{\prime}\left(x^{i}-x^{j}\right)\left(x^{i}-x^{k}\right)\left(d x^{i}\right)^{2}}{\left(x^{i}-a_{1}\right)\left(x^{i}-a_{2}\right)\left(x^{i}-a_{3}\right)\left(x^{i}-a_{4}\right)} \\
(i, j, k \neq) .
\end{aligned}
$$

This metric corresponds to the choice of elliptic type coordinates related to the reduction $o(6, \mathrm{C}) \supset o(2, \mathrm{C}) \times o(4, \mathrm{C})$. If the roots $\left\{a_{j}\right\}$ are unequal they may be chosen as $a, b, 1,0$, respectively. The corresponding four space coordinates are

$$
\begin{gathered}
z^{1}=F \sinh x^{1}, \quad z^{2}=\eta_{1} F, \quad z^{3}=\eta_{2} F, \quad z^{4}=\eta_{3} F, \\
F=\left(\eta_{0}-\cosh x^{1}\right)^{-1}, \quad \eta_{0}^{2}+\eta_{1}^{2}+\eta_{2}^{2}+\eta_{3}^{2}=1,
\end{gathered}
$$

and the choice of coordinates on the sphere $S_{3}$ is

$$
\begin{array}{ll}
\eta_{0}^{2}=\frac{-\left(x^{2}-a\right)\left(x^{3}-a\right)\left(x^{4}-a\right)}{(b-a)(1-a) a}, & \eta_{1}^{2}=\frac{-\left(x^{2}-b\right)\left(x^{3}-b\right)\left(x^{4}-b\right)}{(a-b)(1-b) b} \\
\eta_{2}^{2}=\frac{-\left(x^{2}-1\right)\left(x^{3}-1\right)\left(x^{4}-1\right)}{(a-1)(b-1)}, & \eta_{3}^{2}=\frac{x^{2} x^{3} x^{4}}{a b}
\end{array}
$$

Here

$$
Q=\left[\left[\frac{\left(x^{2}-a\right)\left(x^{3}-a\right)\left(x^{4}-a\right)}{(a-b)(1-a) a}\right]^{1 / 2}-\cosh x^{1}\right]^{-2} .
$$

If some of the roots are equal, this just amounts to a new choice of elliptic coordinates on the sphere $S_{3}: \eta \cdot \eta=1$, see [1]. Here the Riemannian space is $S_{3} \times E_{1}$.

If $a=0$ the metric is

$$
d \hat{s}^{2}=\left(d x^{1}\right)^{2}+\sum_{i=2}^{4} \frac{\left(x^{i}-x^{j}\right)\left(x^{i}-x^{k}\right)\left(d x^{i}\right)^{2}}{\left(x^{i}-a_{1}\right)\left(x^{i}-a_{2}\right)\left(x^{i}-a_{3}\right)} .
$$

The metric corresponds to the choice of elliptic type coordinates related to the reduction $o(6, C) \supset \mathcal{E}(4, C) \supset \mathcal{C}(3, \mathrm{C})$. Here we have assumed for illustrative purposes that $f(z)$ is a third order polynomial although all metrics with $\operatorname{deg} f(z)<3$ correspond to reductions of this type. If all the roots in (4.28) are distinct we may take $a_{1}=a, a_{2}=1, a_{3}=0$. The corresponding choice of four space coordinates is 


$$
\begin{aligned}
& z^{1}=Q^{1 / 2} x^{1}, \quad z^{2}=Q^{1 / 2}\left[\frac{\left(x^{2}-a\right)\left(x^{3}-a\right)\left(x^{4}-a\right)}{a(a-1)}\right]^{1 / 2}, \\
& z^{3}=Q^{1 / 2}\left[\frac{\left(x^{2}-1\right)\left(x^{3}-1\right)\left(x^{4}-1\right)}{1-a}\right]^{1 / 2}, z^{4}=Q^{1 / 2}\left[\frac{x^{2} x^{3} x^{4}}{a}\right]^{1 / 2},
\end{aligned}
$$

where

$$
Q=1 \text { or } Q=\left[\left(x^{1}\right)^{2}+x^{2}+x^{3}+x^{4}-a-1\right]^{-2} \text {. }
$$

(The two choices for $Q$ yield $o(6, \mathrm{C})$-equivalent coordinates.) For general cases of type [3] metrics it is straightforward but lengthy to show that no new coordinates appear. For metrics of type [5] allowing some of the functions $\sigma_{i j}$ to be constant produces systems which run through the various separable coordinates on the sphere $S_{3}$ [1], [2]. Here, with $Q=1$, the Riemannian space is flat.

For metrics of type [4] we can take $\sigma_{i}=x^{i}$ and substitute the metric coefficients into $B_{12,34}=B_{13,24}$ to get

$$
\begin{aligned}
& \sum_{l=1}^{4}\left[\left(x^{i}-x^{j}\right)\left(x^{i}-x^{k}\right)\left(x^{j}-x^{k}\right)\right]^{3} \\
& \quad \cdot\left\{\left(x^{l}-x^{i}\right)\left(x^{l}-x^{j}\right)\left(x^{l}-x^{k}\right)\left(\frac{1}{X_{l}}\right)^{\prime}\right. \\
& \quad-2\left[3\left(x^{l}\right)^{2}-2 x^{l}\left(x^{i}+x^{j}+x^{k}\right)\right. \\
& \left.\left.+\left(x^{l} x^{j}+x^{i} x^{k}+x^{j} x^{k}\right)\right]\left(\frac{1}{X_{l}}\right)\right\}=0
\end{aligned}
$$

where $\{i, j, k, l\}$ is an even permutation of $\{1,2,3,4\}$. These equations have the general solution

$$
\frac{1}{X_{i}}=f\left(x^{i}\right)=\sum_{m=0}^{6} a_{m}\left(x^{i}\right)^{m}, \quad i=1,2,3,4 .
$$

If $a_{6} \neq 0$ then it is always possible to take $a_{0}=0$ and change coordinates according to $x^{i} \rightarrow\left(x^{i}\right)^{-1}$. Pulling out the factor $\left(x^{1} x^{2} x^{3} x^{4}\right)^{-1}$ we see that $X_{i}^{-1}$ can quite generally be taken such that $a_{6}=0$. The metric is then

$$
d s^{2}=\sum_{i=1}^{4} \frac{\left(x^{i}-x^{j}\right)\left(x^{i}-x^{k}\right)\left(x^{i}-x^{l}\right)}{\Pi_{p=1}^{5}\left(x^{i}-a_{p}\right)}\left(d x^{i}\right)^{2} \text {. }
$$

This metric corresponds to the various types of elliptic coordinates possible 
on the sphere $S_{4}$ related to the reduction $o(6, \mathrm{C}) \supset o(5, \mathrm{C})$. If the $\left\{a_{p}\right\}$ are all distinct then the four space coordinates are

$$
\begin{gathered}
z^{i}=Q^{1 / 2} \eta_{i}, \quad i=1,2,3,4, \quad Q=\left(1+\eta_{5}\right)^{-2}, \\
\sum_{i=1}^{5} \eta_{i}^{2}=1 .
\end{gathered}
$$

The coordinates on $S_{4}$ are

$$
\eta_{i}^{2}=\frac{\prod_{j=i}^{4}\left(x^{j}-a_{i}\right)}{\prod_{j \neq i}\left(a_{i}-a_{j}\right)} .
$$

When some of the roots are equal then (4.32) corresponds to one of the various degenerate elliptic type coordinates on $S_{4}$ [3], [4]. (It is interesting to note that the $\left\{x^{j}\right\}$ in these cases are the general cyclidic coordinates in which the Laplace equation separates [4]. We have shown that these cyclidic coordinates correspond to elliptic coordinates on $S_{4}$.)

If $\operatorname{deg} f(z)<4$ the resulting differential form corresponds to various types of elliptic coordinates in flat space which are related to the reduction $o(6, C) \supset \mathcal{E}(4, C)$ [2], [4]. If $\operatorname{deg} f(z)=4$ with distinct roots then

$$
\begin{aligned}
\left(z^{i}\right)^{2} & =Q \frac{\Pi_{j=1}^{4}\left(x^{j}-a_{i}\right)}{\prod_{j \neq i}\left(a_{i}-a_{j}\right)}, \\
Q & =1 \text { or } Q^{1 / 2}=\sum_{i=1}^{4} \frac{\Pi_{j=1}^{4}\left(x^{j}-a_{i}\right)}{\prod_{j \neq i}\left(a_{i}-a_{j}\right)} .
\end{aligned}
$$

(The two choices of $Q$ are $o(6, \mathbf{C})$ equivalent.) If some of the roots are equal to $\operatorname{deg} f(z)<4$ the resulting coordinates are of the form $z^{i}=Q^{1 / 2} \bar{z}^{i}$ where the $\left\{\bar{z}^{i}\right\}$ are separable flat space coordinates and $Q=\left(\sum_{i=1}^{4}\left(\bar{z}^{i}\right)^{2}\right)^{-2}$.

This completes our classification of separable systems for the HamiltonJacobi equation (1.1)(b). Since each of the Riemannian spaces $E_{4}, S_{2} \times S_{2}$, $S_{3} \times E_{1}, S_{4}$ which arise in this classification has constant scalar curvature $R$, it follows from Theorem 2 that all these systems permit $R$-separation for the Laplace equation (1.1)(a).

THEOREM 3. An orthogonal coordinate system permits separation of variables for the flat space Hamilton-Jacobi equation

$$
\sum_{i=1}^{4}\left(\frac{\partial W}{\partial z^{i}}\right)^{2}=0, \quad d s^{2}=\sum_{i=1}^{4}\left(d z^{i}\right)^{2}
$$

iff it permits $R$-separation for the Laplace equation

$$
\sum_{i=1}^{4} \frac{\partial^{2} \Psi}{\partial\left(z^{i}\right)^{2}}=0
$$


Furthermore the metric associated with each such coordinate system $\left\{x^{j}\right\}$ can be written as

$$
d s^{2}=Q d \hat{s}^{2}=Q\left[\sum_{i=1}^{4} h_{i}^{2}\left(d x^{i}\right)^{2}\right]
$$

where $d \hat{s}^{2}$ permits true separation of variables for the Helmholtz equation $\Delta_{2} \Phi=E \Phi$ on one of the manifolds: (1) $E_{4}$, (2) $S_{2} \times S_{2}$, (3) $S_{3} \times E_{1}$, (4) $S_{4}$. All separable systems on these manifolds permit $R$-separation for the Laplace equation.

\section{REFERENCES}

1. E. G. Kalnins and W. Miller, Jr., Lie theory and the wave equation in space time. II: The group SO(4, C), SIAM J. Math. Anal. 9 (1978), 12-33.

2. Lie theory and the wave equation in space time. IV: The Klein Gordon equation and the Poincaré group, J. Mathematical Phys. (to appear).

3. _ Orthogonal separable coordinates for the Laplace-Beltrami operator on the complex four-sphere (preprint).

4. Lie theory and the wave equation in space time. V: $R$-separable solutions of the wave equation, J. Mathematical Phys. 18 (1977), 1741-1751.

5. Lie theory and the wave equation in space time. III: Semi-subgroup coordinates, J. Mathematical Phys. 18 (1977), 271-280.

6. M. Bôcher, Die Reihentwickelungen der Potentialtheorie, Leipzig, 1894.

7. L. P. Eisenhart, Stäckel systems in conformal Euclidean space, Ann. of Math. (2) 36 (1935), 57-70.

8. C. Boyer, E. G. Kalnins and W. Miller, Jr., R-separable coordinates for three-dimensional complex Riemannian spaces, Trans. Amer. Math. Soc. 242 (1978), 355-376.

9. W. Miller, Jr., Symmetry and separation of variables, Encyclopedia of Applicable Mathematics, Vol. 4, Addison-Wesley, Reading, Mass., 1977.

10. P. Moon and D. E. Spencer, Theorems on separability in Riemannian n-space, Proc. Amer. Math. Soc. 3 (1952), 635-642.

11. P. Stäckel, Über die integration der Hamilton-Jacobischen differentialgleichung mittels separation der variabelen, Halle, 1891.

12. L. P. Eisenhart, Separable systems of Stäckel, Ann. of Math. (2) 35 (1934), 284-305.

13. P. Moon and D. E. Spencer, Separability conditions for the Laplace and Helmholtz equations, J. Franklin Inst. 253 (1952), 585-600.

14. L. P. Eisenhart, Riemannian geometry, 2nd printing, Princeton Univ. Press, Princeton, N. J., 1949, pp. 89-92.

School of Mathematics, University of Minnesota, Minneapolis, Minnesota 55455 (Current address of Willard Miller, Jr.)

Current address (E. G. Kalnins): Department of Mathematics, University of Waikato, Hamilton, New Zealand 